

\title{
Las políticas laborales y las relaciones de trabajo durante los gobiernos del Frente Amplio
}

\author{
Labor policies and labor relations during the \\ governments of the Frente Amplio
}

*Fabión Corracedo

${ }^{*}$ Luis Senatore

\section{Resumen}

Las acciones políticas emprendidas en materia laboral por los gobiernos del Frente Amplio configuraron un mapa de relaciones laborales significativamente opuesto al representado en la década de los noventa de flexibilización y desregulación laboral. En efecto, desde 2005 se observa un proceso de fortalecimiento de las institucionales laborales que ha repercutido en los niveles de ingresos y condiciones de vida de gran parte de la población. En concreto, el presente trabajo tiene como cometido describir las políticas laborales y las relaciones de trabajo a la vista de las transformaciones experimentadas durante los gobiernos de Tabaré Vázquez (2005-2010) y de José Mujica (2010-2015).

\begin{abstract}
Political actions undertaken in labor matters by governments of the Frente Amplio configured a map of labor relations significantly opposite to that shown in the nineties by labor flexibility and deregulation. Indeed, since 2005 a process of institutional strengthening labor that has affected income levels and living conditions of much of the population is observed. Specifically, the present work is to describe committed labor policies and labor relations in view of the changes experienced during the governments of Tabaré Vázquez (2005-2010) and José Mujica (2010-2015).
\end{abstract}

Keywords: governments of the Frente Amplio, labor policies, labor relations.

Palabras claves: gobiernos del Frente Amplio, políticas laborales, relaciones de trabajo.

\footnotetext{
* Licenciado en Ciencia Política y estudiante de la maestría en Ciencia Política por la Facultad de Ciencias Sociales de la Universidad de la República.

E-mail: ofcarracedo@gmail.com

** Master of Arts in Philosophy de la Universidad Estatal de Moscú. Docente del Instituto de Ciencia Política de la Facultad de Ciencias Sociales de la Universidad de la República. 


\section{Introducción}

La llegada del Frente Amplio (FA) al gobierno nacional se inscribe en el giro político-ideológico ocurrido en la región. Portando un discurso crítico a los legados del proceso neoliberal y orientado a una nueva estrategia de desarrollo favoreció un rol más activo del Estado en la regulación económica, con políticas de estabilidad macroeconómica (monetarias, fiscales, cambiarias) a la par de una agenda de políticas sociales de inclusión y protección social. Particularmente, las reformas emprendidas en estos diez años en materia laboral marcaron un quiebre respecto a la orientación de las políticas laborales aplicadas durante los noventa de desregulación y flexibilización laboral. Contrariamente a las tendencias que abogan por una mayor descentralización e individualización de las relaciones laborales, al cabo de diez años de gobiernos del FA, se produjo un continuo proceso de fortalecimiento de las institucionales laborales con mayor protagonismo del Estado participando y regulando las relaciones laborales, reinstaurando la negociación colectiva tripartita, desarrollando una política de recuperación y de incrementos salariales, de valorización de la función del Salario Mínimo Nacional (SMN) y produciendo una serie de leyes que vienen a ampliar la protección y los derechos de los trabajadores. La consolidación de las instituciones laborales, en un contexto de crecimiento económico a tasas históricas y de generación de empleo, en complementariedad con otras reformas institucionales, incidieron en la reducción de la pobreza y de la desigualdad.

\section{El papel de las instituciones laborales en términos comparados}

La adopción de los preceptos neoliberales en la región conllevó la flexibilización y la desregulación laboral generando la precarización de las relaciones de trabajo, una mayor informalidad y desigualdad, a través de la reducción de derechos sociales vinculados al trabajo o al empleo, dándole una mayor discrecionalidad a los empresarios sobre la organización de los procesos productivos y las condiciones laborales, librándolos de corresponsabilidad sobre el sistema de protección social, endosándoles todos los riesgos del mercado a los trabajadores o debiendo recurrir a políticas focalizadas compensatorias ante la pérdidas de los ingresos laborales. A su vez, se promovió un Estado aislado de las presiones y demandas sociales, sustrayendo los canales institucionales de regulación del conflicto distributivo e induciendo la despolitización, desmovilización y debilitamiento del sindicalismo.

El auge de gobiernos de izquierda o progresistas abrieron una nueva etapa de estudios sobre las consecuencias del proceso neoliberal. En tal sentido, recientemente el mercado de trabajo ha adquirido nueva centralidad para comprender las dinámicas de integración y exclusión social en la región (Sojo y Pérez Sáinz, 2002) poniéndose de manifiesto el rol que desempeñan las instituciones laborales (existencia o no de 
negociación colectiva, actores sindicales fuertes o débiles, normativas laborales, etc.) en generar o revertir las inequidades sociales (CEPAL, 2010, 2012). En efecto, la dinámica de los mercados laborales influye en varias dimensiones de la desigualdad, sea vía la (re) distribución de ingresos, de las relaciones de poder entre capitaltrabajo, los modos de inserción de los trabajadores al mercado de trabajo y en sus oportunidades de acceso a los sistemas de seguridad social.

Como es sabido, los estudios y debates sobre la desigualdad en la región no son nuevos. Sin embargo, cobraron relevancia en la última década en los análisis comparados de las economías políticas de los países desarrollados. Entre las causas más señaladas del aumento de la desigualdad en dichos países, están: los impactos de la globalización, la apertura comercial y financiera, los procesos de deslocalización de las empresas, los avances técnicos-tecnológicos, las presiones fiscales, los recortes en los niveles del gasto público social, los cambios en la oferta y demanda de mano de obra calificada, etc. Asimismo, mientras gran parte de la literatura sobre redistribución y desigualdad se centró sobre las preferencias individuales o en la agregación de intereses y preferencias de los partidos políticos, se ha comenzado a prestar mayor atención al papel que desempeñan los cambios (o no) en las instituciones laborales reconociendo la necesidad de integrar los estudios de los Estados de bienestar con los estudios sobre el mercado de trabajo (Kenworthy y Pontusson, 2005) o entre los de regímenes de producción y los modelos de bienestar (Schröder, 2009).

En esta línea, en la última década aparece una extensa literatura - sobre todo desde de la variedades de capitalismo y la teoría de la regulación - que intenta dar cuenta de cómo determinados arreglos institucionales inciden de diferente forma en los modos de regulación y coordinación del capitalismo. En tal sentido, surgen resultados concordantes (a nivel teórico y empírico), que permiten identificar tipos ideales de sistemas sociales bien diferenciados reproduciendo características propias históricamente construidas en sus trayectorias de desarrollo. Estos modelos tienen formas de coordinación particulares complejas, pero coherentes entre sus instituciones y las diferentes dimensiones de sus políticas (sociales, económicas, laborales, educativas, etc.). Así, las taxonomías de estas configuraciones ideales van desde una simple presentación dicotómica entre economías liberales de mercado y economías coordinadas de mercado (Hall y Soskice, 2002), pasando por tres (Schmidt, 2005), cuatro (Amable et al., 2008; Boyer, 2005; Ebbinghaus, 2000) e incluso cinco (Amable, 2003). A su vez, estas visiones institucionalistas conviven con aquellas que incorporan las interacciones sociopolíticas sobre la diversidad de sistemas sociales (EspingAndersen y Korpi, 1991; Esping-Andersen, 1993; Korpi y Palme, 2003; Korpi, 2006). A pesar de la diversidad de enfoques teóricos, sorprende el grado de solapamiento entre las distintas de "familias" de países y entre grupos de países donde los mecanismos de reglamentación, así como el grado de intervención del Estado en el mercado de trabajo, varían entre los distintos modelos. 
En un extremo, se sitúa el modelo liberal o residual (Estados Unidos y Gran Bretaña, por ejemplo), que se caracteriza por mercados de trabajo sumamente desregulados y flexibles, para favorecer cambios estructurales rápidos que permitan mantener las ganancias a corto plazo e induzcan a los individuos a su fácil "reciclaje" y desplazarse rápidamente de un empleo a otro (Amable, 2003; Boyer, 2005; Amable et al., 2008). Esto se ve incentivado por una escasa participación del Estado en la protección social bajo el precepto de que las prestaciones sociales no deben disuadir a las personas de la búsqueda de empleo (Palier, 2005). Las relaciones de trabajo se basan en el voluntarismo de las partes configurando un sistema de relaciones laborales descentralizado, las negociaciones se ciñen a nivel de empresas, generando bajas tasas de sindicalización y de cobertura de la negociación colectiva.

En el otro extremo, se ubica el modelo socialdemócrata o escandinavo (Dinamarca, Finlandia y Suecia), nacido de una alianza de clases entre obreros industriales y rurales, unificados en la cúpula por gobiernos socialdemócratas, lograron formar una amplia clase media en torno a los mecanismos de redistribución. Contrariamente a ciertos supuestos, existe una moderada protección del empleo ante la necesidad de hacer frente a la competitividad internacional implicando mayores riesgos para los trabajadores, pero esto se ve compensado por un sistema de protección social amplio y generoso (Amable, 2003 Boyer, 2005; Amable et al., 2008). Cuenta con un sistema consolidado de negociación colectiva de canal único (centralizado y coordinado) sobre un conjunto de cuestiones sociales y económicas con participación institucional sindical en la gestión de los recursos públicos y en las políticas activas de empleo (sistema Ghent), y se articula en base a tasas altas de afiliación sindical y alta cobertura de la negociación colectiva.

En una situación intermedia se ubica el modelo conservador-corporativo y el modelo de los países de sur de Europa o mediterráneo. En el modelo conservadorcorporativo (Austria, Bélgica, Alemania, Holanda), existe una mayor protección del empleo que en el modelo liberal y socialdemócrata, dado que el rendimiento del mercado de trabajo y la situación en el empleo determina los niveles de protección social, en tanto las prestaciones sociales se encuentran ligadas al salario y a las cotizaciones realizadas por los trabajadores y empleadores según las diferentes categorías profesionales financiando los sistemas jubilatorios de reparto (Amable, 2003; Freyssinet, 2007; Palier, 2005). Tiene un nivel medio alto de institucionalización de la intervención sindical en la regulación de las relaciones laborales (participación, intermediación y concertación), así como por una negociación colectiva de estructura muy centralizada en unos casos (Austria) y de carácter sectorial (Alemania) y niveles de sindicalización y cobertura de la negociación colectiva intermedios.

Por último, el modelo mediterráneo (España, Grecia, Italia y Portugal). A pesar de compartir características con el modelo continental (seguridad social en base a las contribuciones), presenta características específicas, como: el sesgo hacia las personas de mayor edad, las pensiones representan la mayor proporción del gasto social y las 
políticas de compensaciones por desempleo están poco desarrolladas (Palier, 2005). El retraso económico deja subsistir un importante sector informal, que junto con el tipo de especialización productiva y una estructura basada en micro y pequeñas empresas hacen que la protección formal de empleo se encuentre centrada en la permanencia en el puesto de trabajo, lo cual genera niveles moderados de gastos en protección social y que ésta se vea orientada al "alivio" de la pobreza y centrada en los retiros (Amable, 2003). Se caracteriza por un pluralismo sindical mayor y una menor institucionalización de su intervención, así como por modelos de negociación más fragmentada y de doble canal (a nivel de empresas y negociación sectorial), pero con cobertura universal de la negociación colectiva.

En cualquiera de los modelos el sostenimiento de un alto nivel de protección social aparece condicionado por la capacidad de mantener un alto nivel de empleo (Palier, 2005:14). Asimismo, con excepción del modelo neoliberal el resto mantienen estructuras institucionales de coordinación entre los empresarios, sindicatos y el Estado. En efecto, dentro de las instituciones del Estado de Bienestar, como lo son las instituciones laborales, los recursos que los actores controlan en las relaciones laborales y sobre los mercados de trabajo, son de importancia clave para su participación en los resultados del conflicto distributivo (Korpi, 2003; 2006). Es así que en el pasado, los niveles de movilización obrera y el acceso al poder de los partidos de izquierda (socialdemocracia), el efecto de los sistemas electorales proporcionales, el grado de corporativismo, la densidad sindical y la centralización de la negociación salarial, constituyeron el determinante principal de la talla y del carácter más o menos redistributivo de los Estados de Bienestar (Bradley et al., 2003; Gourevitch, 2003; Iversen y Soskice, 2006, 2009; Kenworthy y Pontusson 2005; Rueda y Pontusson, 2000).

En efecto, en estos últimos tiempos queda en evidencia que las instituciones laborales son determinantes importantes en las diferencias entre países en sus niveles de desigualdad (Baccaro, 2008). Se constata que aquellos países con sistemas de negociación colectiva con mayor grado de centralización-coordinación y altas tasas de sindicalización se asocian con una menor desigualdad económica (Wallerstein, 1999, Rueda y Pontusson, 2000; Bradley et al, 2003; Pontusson, Rueda y Way, 2002); registrando mayores tasas de empleo y bajas de desempleo con inequidades salariales menores que los sistemas descentralizados y menos coordinados (Aidt y Tzannatos, 2002; Baccaro, 2008; Bradley y Stephens, 2007; OCDE, 1997); generando salarios relativamente más altos para los trabajadores menos cualificados (Aidt y Tzannatos, 2002); todo reforzado con cuán extensa sea la cobertura de los convenios colectivos (Traxler y Brand, 2009).

En síntesis, a pesar de los diferentes abordajes teóricos que atienden la diversidad de los sistemas sociales, se destaca que - contrariamente a las tesis que sostienen la convergencia incondicional hacia el modelo neoliberal - los países aún pueden encontrar soluciones que le son propias en sus trayectorias y modelos de desarrollo. En esta tentativa se ha clasificado a la región como un capitalismo jerárquico en el que 
predominan las grandes empresas (nacionales y extranjeras), con mercados laborales fragmentados, poco regulados, grandes reservas de trabajadores poco calificados y baja densidad sindical (Schneider y Karcher 2010; Schneider 2009; Schneider y David Soskice, 2009). Empero, a pesar de este planteo generalizable para la región latinoamericana, no consideran las particularidades regionales obviando la posibilidad de coexistencia de varios tipos de capitalismos o modelos de desarrollo (Bizberg, 2015; Boschi y Gaitán, 2009). Por lo tanto, se corren ciertos riesgos de extrapolar estas teorías a un contexto de capitalismo periférico caracterizado por una elevada heterogeneidad estructural, con mercados de trabajo segmentados, altos grados de informalidad, donde conviven sectores de alta productividad y elevados niveles de remuneración, con sectores más numerosos de productividad intermedia y baja con trabajadores con niveles reducidos de remuneraciones generando una desigualdad estructural de la distribución de los ingresos.

\section{Legados y trayectoria de la reformas neoliberales en Uruguay}

Como la mayoría de los países de la región, Uruguay enfrentó los embates del proceso neoliberal de reestructuración económica y productiva promovido por los organismos financieros internacionales (FMI, BM, etc.) y sostenidos políticamente por coaliciones políticas y socioeconómicas internas. Sin embargo, la trayectoria de los países ha demostrado que existen distintos grados de liberalización económica siempre dependientes de los arreglos institucionales de cada país (Thelen, 2014). Si bien en Uruguay el proceso liberalización comienza durante la década del setenta y toma mayor empuje a partir de los noventa - durante los gobiernos del Partido Nacional y del Partido Colorado - su impulso fue gradual y de manera heterodoxa a diferencia de la radicalidad adoptada en otros países como Chile o Argentina. La moderación del proyecto de reformas no significó una transformación radical de la matriz de protección social, sino que varias áreas del sistema solo estuvieron sujetas a ciertas recalibraciones (Antía et al., 2013). Esto ha significado que Uruguay mantenga un sistema de protección social - aún con ciertos criterios liberales - con un perfil estatal proteccionista (Martínez Franzoni, 2007) o conservando a resguardo elementos de su estructura de estratificación corporativa (Filguera 2005). Sin embargo, la liberalización alcanzó su mayor despliegue sobre el mercado de trabajo: a) con la constitución de nuevas formas de contratación (tercerizaciones, subcontrataciones, arrendamientos de obra, etc.), con el propósito de esconder o disfrazar relaciones de dependencia bajo la denominación de "trabajo autónomo"; b) con el retiro del Estado de la negociación salarial tripartita -con la evidente descentralización - quedando la determinación salarial y las condiciones de trabajo libradas al libre juego de las partes; y c) a nivel político-institucional donde el Estado asume una actitud pasiva o la no acción como política laboral retrayendo las funciones del Ministerio de Trabajo y Seguridad Social (MTSS) a un papel marginal dentro de la institucionalidad estatal. En suma, las políticas 
de liberalización económica y su impacto sobre la estructura productiva, agudizaron la heterogeneidad y segmentación del mercado de trabajo, lo cual conjuntamente con el debilitamiento de las institucionales laborales, condujeron a una "remercantilización" (Pierson, 2006) de las relaciones de trabajo repercutiendo negativamente sobre los trabajadores y en la organización y representación del movimiento sindical.

\section{Hacia un nuevo paradigma de relaciones laborales}

La situación de partida del primer gobierno del FA era de fuertes restricciones socioeconómicas. La recesión iniciada en 1998 y su corolario en la crisis de 2002, dejaron atrás las supuestas bondades del modelo económico cuyas consecuencias significaron el aumento de la deuda por el $100 \%$ del PBI y su caída en el orden del 11\%, la pérdida del salario real en más del $25 \%$, el desempleo rayó un valor máximo de 19 \% (primer trimestre de 2002), la pobreza se elevó al 40 \% en 2004, con más de 100 mil personas en situación de indigencia, la informalidad como no registro a la seguridad social rayó el 41 \% y la desigualdad del ingreso medida por el índice de Gini alcanzó 45,3. En tal sentido, como parte de la plataforma de gobierno y con el fin de revertir los efectos de la crisis se desplegaron una serie de reformas a nivel institucional y en diferentes áreas de políticas sectoriales de corte distributivo y redistributivo como: a) la tributaria que incorpora un régimen de tasación progresivo; b) la reforma de la salud ampliando su cobertura a una mayor parte de la población; c) en la seguridad social con la extensión y creación de prestaciones; y d) la reinstitucionalización de la negociación colectiva tripartita; e) a la par de políticas de transferencias de rentas (condicionadas o no), tendientes a reducir la pobreza y la exclusión social.

Particularmente en materia laboral, el gobierno de Tabaré Vázquez (2005 - 2010) se caracterizó por su impulso reformista, mientras que el de José Mujica (2010 2015) tuvo por delante el desafío de asentar la nueva institucionalidad laboral. En concreto, al cabo de una década de gobiernos del FA, existe un quiebre políticoideológico e institucional en la orientación de las políticas laborales y relaciones de trabajo respecto a las implementadas durante la década de los noventa. En esta línea, el Estado retoma mayor protagonismo en materia políticas laborales que se ve reflejado: a) en la jerarquización del rol del MTSS fortaleciendo sus capacidades institucionales, readquiriendo iniciativa política y capacidades de regulación; b) con la restauración de los Consejos de Salarios (CCSS), como mecanismo de negociación colectiva tripartita, incorporando a sectores históricamente excluidos como el rural y el servicio doméstico, y haciéndola extensiva bipartitamente a toda la administración pública; c) desarrollando una política activa de recuperación y crecimiento salarial, con diferenciales para los sectores con salarios más sumergidos y de valorización del papel del SMN; y d) una alta producción en materia de leyes de protección de los trabajadores (individuales y colectivos) y que amplían sus derechos sociales. Por otra parte, se fortalecieron las políticas activas de mercado de trabajo, sobre todo para 
aquellos sectores más vulnerables y con mayores problemas de inserción laboral (jóvenes, mujeres, discapacitados, etc.) y se crearon iniciativas de respaldo a proyectos productivos de asistencia a empresas autogestionadas o de unidades productivas recuperadas por los trabajadores.

A pesar de la situación de partida, los gobiernos del FA contaron con ciertas condiciones favorables para llevar adelante su proyecto político. En el plano económico, desde 2004 Uruguay comienza una etapa de constante crecimiento económico (aún crisis mundial de 2008-09 mediante), favorecido por un contexto económico internacional de incremento de los precios de los commodities, por el aumento de la Inversión Extranjera Directa (IED) y el crecimiento de la demanda del mercado interno que impulsaron un mayor dinamismo del mercado de trabajo. En el plano político, ambos gobiernos del FA contaron con mayorías parlamentarias en ambas cámaras dándole un amplio margen de acción (por ejemplo, el sancionar leyes ordinarias y especiales) para llevar adelante sus reformas político-institucionales. En lo societal, los vínculos del partido de gobierno con el movimiento sindical permitieron configurar un escenario de cooperación o de "apoyo mesurado" para la consecución del proyecto político de los dos gobiernos del FA. Esto último resultó significativo, particularmente durante el período de Tabaré Vázquez, en tanto era la primera experiencia del FA en el gobierno nacional, donde tuvo que hacer frente a las demandas del movimiento sindical, debiendo guardar a su vez una mirada sistémica de los intereses de la sociedad. A la inversa, el sindicalismo tuvo el desafío de enfrentarse a un gobierno de un partido "amigo", pero defendiendo, al mismo tiempo, su condición clasista y su autonomía respecto a los partidos políticos.

En el marco del llamado al Diálogo Social, el primer gobierno del FA conformó ámbitos de deliberación, participación y de negociación de políticas en materia laboral. Por un lado, se creó - sin norma expresa - el Compromiso Nacional para el Empleo, los Ingresos y las Responsabilidades y se reglamentó el Consejo de Economía Nacional $(C E N)^{1}$ intentando simular ensayos europeos o regionales (el CEDES, por ejemplo) ${ }^{2}$ de involucrar a los principales actores sociales en la orientación de las políticas de desarrollo. Sin embargo, ambas instancias tuvieron una breve proyección. El primero cesó con el retiro de los empresarios frente a la aprobación de normas laborales que no les eran favorables $y$, en el segundo caso, se restringió a meros pronunciamientos referidos a la creación de empleos y reducción de la informalidad. A diferencia de lo sucedido a nivel del diálogo social, allí donde se daría la puja (re)distributiva, existió una jerarquización de los temas adquiriendo otra densidad política y socioeconómica, mayor verticalidad e intervención del Estado en la regulación de las relaciones laborales constituyendo una serie núcleos neocorporativos, intermediando y reordenando las relaciones capital-trabajo (Carracedo, 2010).

Entre las medidas políticas más importantes tomadas por el primer gobierno del FA se encuentra la reinstitucionalización de los Consejos de Salarios (CCSS), como mecanismo de negociación colectiva tripartita. Asimismo, se instituyó el Consejo 
Superior Tripartito (CST), con la finalidad de clasificar los distintos grupos y subgrupos de negociación por sectores o ramas de actividad y se creó el Consejo Bipartito (CB) cubriéndose casi todos los organismos e instituciones del Estado en distintos grupos y niveles de negociación: superior, sectorial y por cada organismo, tanto a nivel nacional como departamental. A su vez, se conformó en el Consejo Tripartito Rural (CTR) y se anexaron a los CCSS el sector del servicio doméstico. Lo relevante de esto último es que hasta entonces los salarios eran fijados por decreto del Poder Ejecutivo e incorpora a la negociación colectiva tripartita un segmento poblacional tendencialmente vulnerable, con altos grados de informalidad, de bajos ingresos, sometida a una fuerte dependencia y discrecionalidad en las relaciones patrón-empleado, en entornos físicos laborales aislados, con dificultades para ejercer sus derechos (como a la sindicalización) y expresar sus reivindicaciones. A la par, se procedió a jerarquizar institucionalmente y a recomponer las capacidades estatales del MTSS - sumamente reducidas durante los noventa - invirtiendo en infraestructura física y en recursos humanos, para mejorar su capacidad regulatoria y como rector de la nueva orientación de las políticas laborales.

Los dos gobiernos del FA retomaron una tradición - olvidada durante la década de los noventa - de producción de normas de protección individual de los derechos de los trabajadores, pero cortaron el abstencionismo en materia de derecho colectivo que dejaba un amplio margen a la creación doctrinal y jurisprudencial (Ermida, 2008; Rossi, 2006). En efecto, desde 2005 a la fecha, se llevan aprobadas más de sesenta leyes con componentes laborales y vinculadas a la seguridad social, entre las que se destacan: la ley que modifica el plazo de prescripción de los créditos laborales; la que abrevia el plazo de los juicios laborales; la ley de tercerizaciones que regula la descentralización empresarial estableciendo las responsabilidades (subsidiarias y solidarias, los aportes a la seguridad, social, etc.) entre las empresas que toman mano de obra tercerizada; la ley que regula el trabajo doméstico; la que establece el régimen laboral del sector rural; y las leyes que lo hacen para el personal dependiente en varias otras ocupaciones (gastronomía, hotelería, enfermería, etc.); la ley de acoso sexual en el ámbito laboral; la ley de subsidio maternal y paternal; la de no discriminación a los afrodescendientes; la prohibición de solicitar a las mujeres un test de embarazo para acceder a un empleo; la ley de responsabilidad penal empresarial, etc.

En el plano del derecho colectivo, se produjo un giro abandonando la práctica de autorregulación y autonomía de los actores de las relaciones laborales. Esta tendencia se tradujo en los decretos que regulan la ocupación de los lugares de trabajo (en sector privado y público), como modalidad del derecho de huelga ${ }^{3}$, la ley que tutela la libertad sindical ${ }^{4}$, la ley de negociación colectiva para el sector público y la ley que crea el Sistema de Negociación Colectiva del sector privado. Esta última ley no sustituye a los CCSS, sino que coordina institutos ya creados y constituye el marco institucional para desarrollar la negociación colectiva a distintos niveles. Se establece al CST como órgano de coordinación y gobernanza atribuyéndole, entre otras funciones, la clasificación de los grupos de negociación por rama de actividad, asesorar el Poder 
Ejecutivo en casos administrativos, pronunciarse en temas vinculados a los niveles de negociación tripartita y bipartita, etc. Le siguen los CCSS a nivel tripartito como pilar de las relaciones laborales, luego la negociación bipartita a nivel de rama de actividad y, por último, la negociación por empresa, con la particularidad que las negociaciones a niveles inferiores no pueden disminuir los mínimos acordados en los convenios colectivos a un nivel superior. A su vez, deja abierta la vigencia de los convenios colectivos hasta que otro lo sustituya (su ultraactividad) e introduce las formas y mecanismos de prevención y solución de conflictos (cláusula de paz), donde las partes se comprometen a no realizar medidas de fuerza durante la vigencia del convenio colectivo, exceptuandolas medidas sindicales que sean de carácter nacional.Porúltimo, entre los aspectos más innovadores, a diferencia de las anteriores etapas, el Poder Ejecutivo ya no cuenta con el monopolio de convocar los CCSS, siendo ahora potestad de cualquiera de los actores (gobierno, empresarios y/o movimiento sindical) y pierde cierto grado de intervención o regulación sobre la determinación salarial al sustituirse las antiguas "pautas" por "lineamientos" salariales y lo convenido tripartitamente ya no requiere de un decreto del Poder Ejecutivo para su homologación.

\section{Los impactos de las reformas laborales en los actores}

La reinstalación de la negociación colectiva trajo consigo nuevas reglas de juego para los actores de las relaciones laborales. Al cabo de diez años, los CCSS como eje central de la institucionalidad laboral, no sólo establecieron el marco de regulación y coordinación entre gobierno, PIT-CNT y las cámaras empresariales, sino que han cumplido una función política democratizando las relaciones de trabajo, una función económica al intervenir en la distribución primaria del ingreso y una función social, canalizando, administrando y dirimiendo el conflicto surgido de la puja distributiva capital-trabajo; incluso incorporando en los convenios colectivos cláusulas que vienen a extender derechos ya consagrados por la legislación (como aconteció en algunas ramas de actividad donde se otorgaron regímenes especiales de licencia maternal y paternal) (Carracedo y Senatore, 2013).

Como en otras etapas, la negociación colectiva de carácter centralizado (sectorial y/o rama de actividad) reestructuró de igual modo la acción y organización colectiva de las cámaras empresariales y del movimiento sindical. Tanto empresarios (aunque sin cifras exactas) y PIT-CNT, vieron recomponer sus respectivas filas. La cercanía ideológica del partido de gobierno, las normas que protegen y garantizan la libertad sindical, y ciertos incentivos selectivos y colectivos institucionales (ámbitos especializados de gestión política tripartita) permitieron la reconstitución del movimiento sindical. A las reformas realizadas en el plano laboral, se sumó la adscripción de representantes del movimiento sindical a segmentos de corte neocorporativo en áreas matriciales del Estado como en la salud, la educación, en la seguridad social y en la regulación del mercado de trabajo ${ }^{5}$, que le confirieron al PIT-CNT status de actor sociopolítico, 
atributos de poder, de representación y representatividad; bienes materiales y simbólicos; y consolidar su organización al atraer nuevos y fortalecer viejos sindicatos. En efecto, al cabo de diez años el PIT-CNT vio engrosar sus filas con el crecimiento de su densidad sindical, pasando en 2005 de 120 mil a cerca de 400 mil afiliados en 2015.

Gráfico 1. Afiliados cotizantes a los Congresos del PIT-CNT (1985 - 2015)

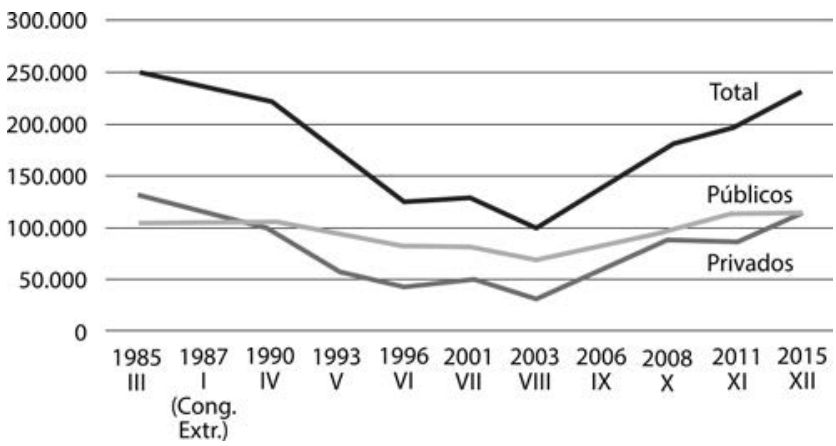

Fuente: Elaboración a partir de los datos suministrados por la Comisión de Poderes de los Congresos del PIT-CNT

El fortalecimiento del movimiento sindical le confirió recursos de poder y capacidad de veto en el sistema político. Durante el gobierno de Tabaré Vázquez, el PIT-CNT logró mantener su potencial de control y mediación de la estructura sindical conteniendo las acciones free riding, ordenando las relaciones entre cúpula y las"nuevas bases"sindicales (más heterogénea y más joven, sin acervo sindical, menos politizada y con objetivos cortoplacistas) y acotando las acciones de los sectores minoritarios más radicalizados, prevaleciendo las posiciones tendientes a compromisos y actitudes responsivas ante lo que era la primera experiencia de la izquierda en el gobierno (Carracedo y Senatore, 2013 $)^{6}$. Sin embargo, durante el gobierno de José Mujica, dentro de la interna sindical se dieron una serie de confrontaciones asociadas a cambios en la correlación de fuerzas entre aquellos sectores más cercanos al gobierno - pero pronunciando su autonomía de clase - y quienes asumen posiciones más radicalizadas (Carracedo y Senatore, 2013). Estas diferencias a la interna del PIT-CNT se vienen procesando desde hace tiempo, pero encontraron su punto más alto durante el gobierno de Mujica donde los sectores más críticos al corte y al alcance las políticas del FA lograron mayor representación (Méndez y Senatore, 2012). El aumento de la conflictividad se debió a cierto desdibujamiento de los lazos de afiliación cruzada entre tendencias políticas o agrupamientos internos sindicales y sectores políticos del FA, sumándose el pasaje de muchos dirigentes de larga trayectoria sindical a cargos de gobierno (significando una pérdida en el acervo y transferencia de la experiencia y cultural sindical). A su vez, el gobierno de José Mujica no contó con el impulso reformista del primer gobierno del FA, cambiando los temas y los objetos de intercambio político con el PIT-CNT, 
coadyuvado por un estilo de conducción presidencial y gestión de gobierno de Mujica, que en ocasiones alimentó tensiones, principalmente con los sindicatos del sector público.

Por otra parte, la nueva normativa laboral fue la causa mayor de enfrentamientos entre el gobierno y empresarios. Se acusó a los gobiernos del FA de perder neutralidad, de torcer la"balanza" en favor de los sindicatos y no tomar en consideración su posición y sus demanda, sosteniendo que la nuevas leyes laborales, junto con la reincorporación de la negociación colectiva tripartita, serían elementos que aumentarían las rigideces del sistema provocando desempleo, aumento de la inflación, caída de la productividad, de la competitividad, etc., repercutiendo todo sobre el nivel general de la economía. Lo cierto es que la nueva normativa laboral viene a romper la prevalencia empresarial, creando un marco de garantías a las negociaciones intentando minimizar relativamente la asimetría natural entre capital y trabajo. Por ello, las normas más objetadas por el empresariado, fueron aquellas que limitaban la libre disposición de mano de obra y/o evasión de las responsabilidades patronales como factor de reducción de costos (ley de tercerizaciones) o la permisividad de acciones antisindicales (ley de libertad sindical).

El punto más álgido de tensión entre gobierno y cámaras empresariales, sucedió durante el gobierno de Mujica, cuando éstas interpusieron ante la OIT una queja sobre la nueva ley de negociación colectiva, por entender que va en contra de los convenios № 87 sobre libertad sindical y los convenios № 98, № 144 y № 154 sobre negociación colectiva y consultas tripartitas. A instancias de la OIT y sus recomendaciones se realizaron varias consultas y negociaciones entre el gobierno, el PIT-CNT y las cámaras empresariales con el fin de consensuar un proyecto modificativo a la ley. Tras un frustrado acuerdo en Ginebra y una visita técnica de la OIT en Uruguay en 2011, la etapa de negociaciones con el PIT-CNT y las cámaras empresariales se dio por finalizada en setiembre de 2012. Así, tras dos años de negociaciones, el Poder Ejecutivo resolvió enviar al Parlamento cambios a la ley según las observaciones del Comité de Libertad Sindical (CLS) de la OIT, que aún se encuentra en trámite parlamentario y se ha mantenido como frente de conflicto durante el tercer gobierno del FA amenazando las cámaras empresariales de interponer nuevamente una queja ante la OIT, lo cual incluiría a Uruguay en una "lista negra" colocándolo en pie de igualdad con otros países donde no se respetan los derechos laborales, las libertades sindicales o que registran asesinatos de los dirigentes sindicales. Sin embargo, los empresarios decidieron no reactivar la queja o dejar en suspenso teniendo en cuenta que el presidente Tabaré Vázquez fue convocado como invitado de honor para abrir la 106 Conferencia de la OIT en junio de 2017 y, donde al mismo tiempo, se comprometió a solicitarle al organismo internacional asesoramiento técnico para continuar el proceso de negociación entre representantes de los trabajadores, empresarios y el Estado. En concreto, es un tema abierto, no resuelto donde será difícil lograr un consenso dado que los empresarios procuran que la negociación colectiva se realice a nivel bipartito ahí donde la fortaleza 
de los sindicatos y trabajadores es menor, y que se regule el derecho huelga (yendo en contra de una larga tradición uruguaya de autoregulación del conflicto), debilitando así los mecanismos reivindicativos de los sindicatos. Por su parte, el PIT-CNT rechazan de plano cualquier modificación a la ley de negociación colectiva y la regulación de la huelga entendiendo que sería un retroceso en los derechos adquiridos de todos los trabajadores.

A pesar que la conflictividad laboral fue en aumento durante los dos gobiernos del FA, se mantuvo por debajo al período anterior a 2005 de no funcionamiento de los CCSS. Como se observa en el gráfico, los picos de conflictividad se encuentran ligados a determinadas coyunturas. En primer lugar, es de esperar una mayor conflictividad al inicio de cada gobierno dado que se presenta el presupuesto quinquenal y cuando se realiza la última rendición de cuentas. En segundo lugar, también sucede en los momentos previos a las rondas de negociación salariales en los CCSS, para posteriormente descender al final de cada gobierno.

Gráfico 2. Índice de conflictividad laboral promedio anual (Base 2000=100)

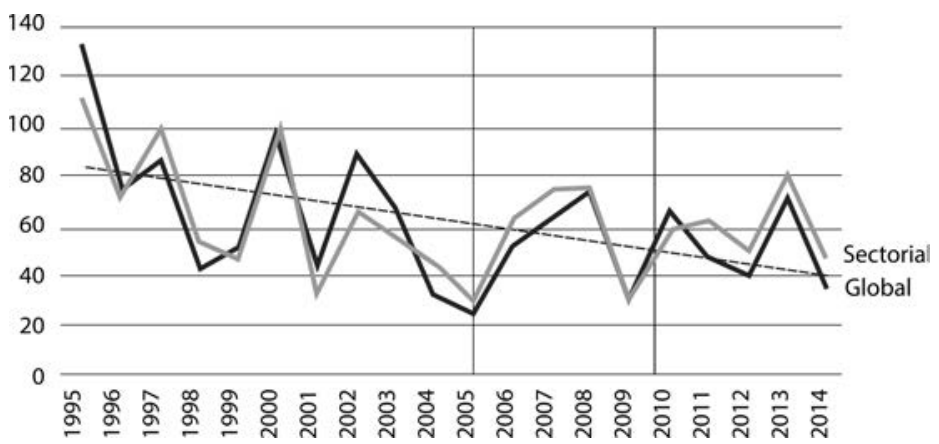

Fuente: Elaboración a partir de datos del Instituto de Relaciones Laborales de la Universidad Católica

\section{Los resultados del fortalecimiento de las instituciones laborales}

Sin embargo, allí donde se daba la puja distributiva los resultados fueron más positivos. Entre 2005 y 2015 se realizaron cinco rondas de negociaciones en los CCSS. La dinámica para aprobar los convenios colectivos es someterlos a votación entre el gobierno, empresarios y sindicatos. En concreto, en dicho período se destaca el número de convenios colectivos aprobados de forma consensuada tripartitamente promediando el $86 \%$, luego los acuerdos en mayoría cercanos al $10 \%$, destacándose que en varias oportunidades los representantes del gobierno votaron casi de igual modo en favor de los empresarios, como de los representantes de los trabajadores.

Luego de diez años de funcionamiento de los CCSS y tras una política de recupe-ración y de crecimiento salarial, los salarios reales medios registraron un 
aumento acumulado cercano al 52\% permitiendo sobrepasar los niveles previos a la crisis de 2002, y manteniendo un línea de aumento continuo del SMN, pasando de $\$ 2.050$ pesos en 2005 , hasta alcanzar los $\$ 10.000$ pesos en 2015 , representando un incremento nominal de $390 \%$ y más de $130 \%$ en términos reales. Aunque los términos negociados en los CCSS se centran principalmente sobre el salario, progresivamente se incorporaron otras temáticas en los contenidos de los convenios colectivos. Al presente, el $67 \%$ de los convenios colectivos incorporan una cláusula de género de los cuales el $44 \%$ incorpora la normativa vigente y 52 \% una cláusula especifica (MTSS, 2014). Al mismo tiempo hay un aumento de las cláusulas relativas a garantizar la paz laboral (77\%), a través de mecanismos de prevención de conflictos y los que incorporan cláusulas de salvaguarda (75\%), que permiten redefinir lo pactado en los convenios ante una situación adversa de la economía.

En otro plano, los avances en materia de seguridad social y de normas de protección de social, según el Índice Global de los Derechos elaborado por la Confederación Sindical Internacional (2014) que evalúa los países donde los derechos de los trabajadores están mejor protegidos, sitúa a Uruguay a la altura de países como Alemania, Dinamarca, Finlandia, Noruega, Suecia o Francia, entre otros. Asimismo, la incorporación de los trabajadores rurales y del servicio doméstico a la negociación colectiva tripartita y su ampliación en el sector público incidieron en el aumento de la densidad sindical y en el nivel de cobertura de la negociación colectiva. En efecto, mientras que la tasa de sindicalización durante los noventa, en promedio no superaba el 20 \%, en 2016 se elevó al 31 \% sobre el total de los asalariados, lo cual en términos comparados coloca a Uruguay, por encima de países como Alemania (25\%), España (16\%), Reino Unido (25\%) o lejos de Chile (14\%). En tanto, el principio de universalidad erga omnes de los convenios colectivos permitió que la cobertura de la negociación colectiva pasara del $20 \%$ a prácticamente al $90 \%$ de los asalariados, lejos de Chile (11\%) y por arriba de Dinamarca (83\%) o cercano a los valores de Francia (90\%), de Finlandia (93\%) o de Suecia (93\%).

El mercado de trabajo es el principal vínculo con el sistema de seguridad y protección social, siendo un ámbito específico para que el crecimiento económico se vea reflejado en la reducción de la pobreza y de la desigualdad. Por lo tanto, si bien el crecimiento económico es una condición necesaria, no es suficiente para la generación de empleo, el descenso del desempleo y de la informalidad, y para lograr la reducción de la pobreza o que conlleve mejoras en estructura distributiva. En efecto, entre 1991 y 1998 el crecimiento económico tuvo una tasa acumulada de 4,3 \% con una tasa de desempleo mínima de 8,3\% en 1993, un nivel de pobreza promedio de $18 \%$ y el índice de Gini se situó en 43,8, al final del período. En cambio, al cierre del gobierno de Mujica, el PBI lleva más de una década de crecimiento ininterrumpido a una tasa promedio de 5,5 \% (2004-2014), y a diferencia del período anterior, conllevó una mejora significativa de los indicadores del mercado de trabajo (alcanzando registros históricos en 2011), cerrando al 2015 con niveles de ocupación de 60 \% y de participación laboral de 
$64 \%$, con una tasa de desempleo de $7,5 \%$ y un nivel de no registro a la seguridad social de $25 \%$, coincidiendo con un nuevo descenso de la pobreza a 9,7\%. Todo esto estuvo acompañado por una reducción de la desigualdad expresada, ya sea a través del índice de Gini alcanzado un registro de 38,1 en 2014, ya sea considerando la relación entre el primer decil de ingresos respecto del ingreso medio de aquellos ubicados en el último decil (ratio D1/D10), mostrando su disminución de 19 en 2004 a 12 en 2013, o ya sea por una mayor participación del salario en el PBI, pasando de representar en 2005 el 28,6\% al 39,9\% en el 2011 (Burdin, et al., 2014). En síntesis, como se puede observar en el siguiente gráfico los niveles de desigualdad tuvieron un pronunciado descenso a partir de 2010 y fueron acompañados por una reducción de las desigualdades salariales.

Gráfico 3. Evolución de la desigualdad y la desigualdad de ingresos salariales, 2001-2013

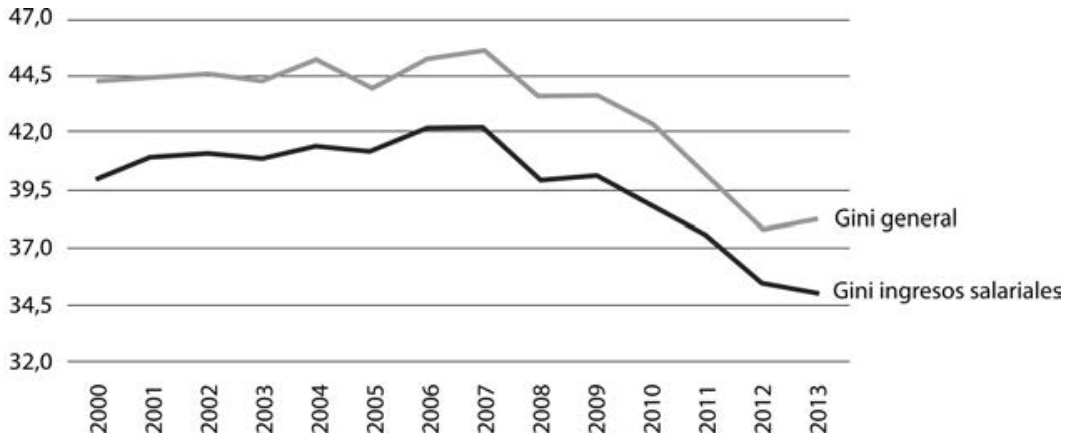

Fuente: INE y Amarante, Arim, Yapor (2015)

Las reformas en materia laboral no solo repercutieron sobre el ámbito estricto del mercado de trabajo, sino que tuvieron cierto impacto sistémico. En términos de la relación salarial (Boyer, 1998) o de complementariedades institucionales las instituciones no son estáticas e interactúan en un marco más amplio, la influencia o la existencia de una institución - o la forma particular que toma en un dominio determinado - refuerza la presencia, el funcionamiento o eficiencia de otra institución en otro dominio (Aoki, 1994; Hall y Sosckice, 2001; Amable, 2003).

La reinstitucionalización de la negociación colectiva y el fortalecimiento del salario mínimo jugaron un papel relevante sobre la estructura salarial, sin embargo, en interacción con otras reformas político-institucionales, permitieron un descenso de la informalidad. En primer lugar, el fortalecimiento de la capacidad inspectiva del MTSS (retomando la iniciativa en seguridad social), con las acciones realizadas por el Banco de Previsión Social (BPS) y los cruces de información con la Dirección Nacional Impositiva (DGI), permitieron el aumento del número de personas registradas en el BPS, pasando de 916.147 en 2004 a 1.482 .933 puestos cotizantes en 2013. A ello se 
agregan los incentivos que generaron los CCSS instituidos para el sector rural y del servicio doméstico regulando los salarios y las condiciones de trabajo de sectores históricamente postergados con niveles altos de informalidad. Por otra parte, la creación del Sistema Nacional Integrado de Salud (SNIS) en 2007, que amplía la cobertura de salud a la familia (hijos y eventualmente cónyuge) de los trabajadores que realización cotizaciones a la seguridad, puede haber incidido en que los trabajadores regularizaran su situación en "negro" ante sus empleadores (Miranda et al., 2014; Amarante et al., 2015). Por último, en lo que refiere a la informalidad, la reforma en el monotributo y la creación del monotributo social del Ministerio de Desarrollo Social (MIDES) amplió la adhesión de los trabajadores independientes al sistema de seguridad social. En suma, la caída del no registro a la seguridad social, principalmente sobre los asalariados y la baja del subempleo, permitieron que el empleo sin restricciones haya crecido del 55 \% en 2004 a $73 \%$ en 2014.

En síntesis, la reinstitucionalización de la negociación colectiva de carácter sectorial y mayor coordinación en la determinación salarial, la política expresa de incrementos del SMN con su potencial distributivo y sirviendo como nivel referencia para los trabajadores del sector informal y el aumento de la formalización tuvieron incidencia en la reducción de la pobreza y de la desigualdad (Alves, et al., 2012; Amarante et al., 2015; Perazzo et al., 2013).

\section{Hacia un mayor protagonismo de las políticas activas de empleo}

El crecimiento experimentado en esta década conjuntamente con la generación de empleo y bajas tasas de desempleo develaron las dificultades de armonizar la demanda con la oferta laboral ante la insuficiencia de cualificaciones de la fuerza de trabajo. En tal sentido, se impulsaron con mayor énfasis políticas activas del mercado de trabajo, principalmente las que refieren a la capacitación y formación profesional de los sectores que presentan mayores problemas de inserción al mercado de empleo: jóvenes, mujeres, trabajadores poco cualificados, vulnerables o en situación de pobreza, etc. En esta línea se creó el INEFOP, como persona de derecho público no estatal y de conformación tripartita, como referente principal en la ejecución de las políticas de capacitación y formación profesional con el fin de mejorar la inserción laboral y empleabilidad de los trabajadores, a la vez atendiendo determinados requerimientos de las empresas. En paralelo, dentro del reordenamiento institucional se formó en la órbita del MTSS la red de Centros Públicos de Empleo (CEPE), para descentralizar y llevar a nivel de territorio servicios de información, orientación e intermediación laboral. Intentando enmarcar distintas realidades referidas al empleo y la formación profesional se convocó al Diálogo Nacional por el Empleo (2012-2013) 7 . Entre los principales ejes temáticos se incorporó la problemática de inserción laboral de los jóvenes. Si bien, el desempleo entre menores de 25 años descendió desde el 
$28 \%$ en 2006 al $19 \%$ en 2014, aún se encuentra lejos de la tasa de desempleo global. En tal sentido, se creó en 2010 la Unidad de Empleo Juvenil dentro del MTSS, con el fin de intervenir sobre el déficit de las políticas públicas en materia de juventud. El corolario de dicha experiencia fue la presentación y aprobación por unanimidad en el Parlamento de la Ley de Empleo Juvenil (No 19.133). Entre sus disposiciones establece una serie de subsidios y beneficios tributarios en distintas modalidades de contratación, haciendo centro en el fomento de las prácticas de capacitación y de formación. Una modalidad está dirigida a la primera experiencia laboral, en el sector público o sector privado, para jóvenes de 15 a 24 años. Otra, para jóvenes hasta 29 años, egresados de las diferentes opciones educativas y formación profesional, para atender la práctica laboral vinculada a su titulación. Otra, implica mecanismos de trabajo protegido para personas menores de 30 años, desempleados y pertenecientes a hogares en situación de vulnerabilidad. Así también, se busca fomentar los emprendimientos juveniles, dirigidos por jóvenes o cuya dirección esté compuesta por un $51 \%$ de jóvenes, entre 18 y 29 años y la antigüedad del emprendimiento no sea mayor a 5 años.

En otro plano, en sintonía con los preceptos del presidente José Mujica, se creó en 2011 el Fondo de Desarrollo Social (FONDES) conformado por el 30 \% de las utilidades del Banco República (BROU), para la financiación de emprendimientos autogestionados y para empresas recuperadas por los trabajadores, diferenciándose de aquellos fondos que apoyan a las micros y pequeñas empresas. Su objetivo es sostener económicamente proyectos productivos que carecen de poco apoyo a nivel crediticio y por parte de las políticas públicas. Ante ciertas críticas de los empresarios y de los partidos de oposición respecto al destino de los recursos económicos y la viabilidad de los proyectos, en 2015, se promulgó la Ley № 19.337 (difiriendo en algunos aspectos con la idea o proyecto inicial del presidente MUJICA), con la intención generalizada de institucionalizar el FONDES que será administrado por dos particiones: la Agencia Nacional de Desarrollo Económico (ANDE) y el INACOOP. Estos fondos estarán dirigidos a cooperativas en distintas modalidades, empresas autogestionadas por sus trabajadores y emprendimientos de la economía social y solidaria.

\section{A modo de síntesis}

A pesar de ciertos malos augurios iniciales por parte de los empresarios y de los partidos de la oposición que sostenían que la negociación colectiva tripartita, la política de crecimiento salarial y la expansión de normas de protección a los trabajadores conllevarían efectos negativos en el dinamismo del mercado de trabajo y afectaría en los niveles de crecimiento económico, al cabo de diez años de gobiernos del FA, se observa un escenario de fortalecimiento de las instituciones laborales en complementariedad a otras reformas político-institucionales - repercutiendo en 
la mejora de varios indicadores socioeconómicos posicionando al país entre los de menor nivel de pobreza y desigualdad de la región.

Las reformas institucionales y las acciones políticas implementadas permiten afirmar que nuestro país se encuentra en un proceso afianzamiento de un nuevo paradigma en materia de políticas y relaciones laborales. En efecto, tomando las principales dimensiones analíticas para caracterizar los sistemas de relaciones laborales, en nuestro caso se evolucionó de un modelo liberal (flexibilización laboral, descentralización de la negociación colectiva con baja cobertura y densidad sindical, rasgos todos ellos presentes en el periodo 1992-2004), a otro, que se encuentra a medio camino del régimen continental-corporativo y el socialdemócrata, pero con ciertas especificidades del modelo mediterráneo (Carracedo y Senatore, 2016). El funcionamiento y la estructuración de la negociación colectiva a nivel sectorial, es coordinado horizontalmente por el Consejo Superior Tripartito (gobierno, cámaras empresariales y PIT-CNT). Asimismo, el efecto erga omnes de los convenios colectivos genera una coordinación vertical que obliga al cumplimiento de los acuerdos celebrados en los CCSS tanto a los trabajadores, como a los empresarios. Por otra parte, un gobierno de filiación socialista, la presencia de un partenariat partidario-sindical, la existencia de una central sindical única (con representación cuasi monopólica partidaria y de los trabajadores) y el grado de centralizacióncoordinación de la negociación colectiva, son características que se asemejan al régimen socialdemócrata. Por el contrario, que las prestaciones sociales tengan un alto grado de dependencia del nivel de empleabilidad y estatus laboral, son rasgos que lo colocarían en el régimen conservador-corporativista. Por otro lado, la alta protección al empleo centrada en la conservación del puesto de trabajo, la predominancia de las micro y pequeñas empresas, y aún la alta informalidad, es un rasgo característico del modelo mediterráneo (Carracedo y Senatore, 2016).

Existen trabajos basados en comparaciones nacionales, principalmente a nivel europeo, que analizan los impactos de las instituciones laborales en los rendimientos y equilibrios macroeconómicos relacionados con la determinación salarial a través de la negociación colectiva. A pesar de la continuidad de los debates al respecto, existe evidencia que en países con larga tradición y continuidad de la negociación colectiva (Alemania, Austria, Dinamarca, Finlandia, Suecia, etc.), su funcionamiento no ha sido pernicioso sobre la actividad económica, incluso con efectos positivos creando escenarios de cooperación y previsibilidad para los principales actores de las relaciones laborales, internalizando externalidades negativas, afrontar y adaptarse a entornos más competitivos, actuar en la generación empleo y sus efectos comprobados en la reducción de la desigualdad.

Si bien, la reinstitucionalización de la negociación colectiva tripartita no afectó el crecimiento económico experimentado en la última década y, como se observa, tuvo incidencia en la extensión de la protección social y en la reducción de la desigualdad, se presentan nuevos desafíos ante un contexto de menor dinamismo económico y a 
causa de ciertos factores estructurales (productivos y del mercado de trabajo), que pueden afectar dinámica a futuro de la negociación colectiva.

Al cierre de este artículo se cumplen un poco más de dos años de un nuevo gobierno del presidente Tabaré Vázquez y el tercero del FA logrando nuevamente de manera consecutiva mayorías parlamentarias. Sin embargo, las condiciones económicas ya no aparecen tan favorables como en la pasada década. El enlentecimiento del crecimiento de la economía mundial y de los principales socios comerciales de la región (Argentina y Brasil), la caída de los precios y de la demanda de commodities, hacen que la economía uruguaya entre en una etapa de menor dinamismo previéndose para los próximos años tasas de crecimiento del PBI del orden del $2 \%$, a lo cual se agrega un déficit fiscal
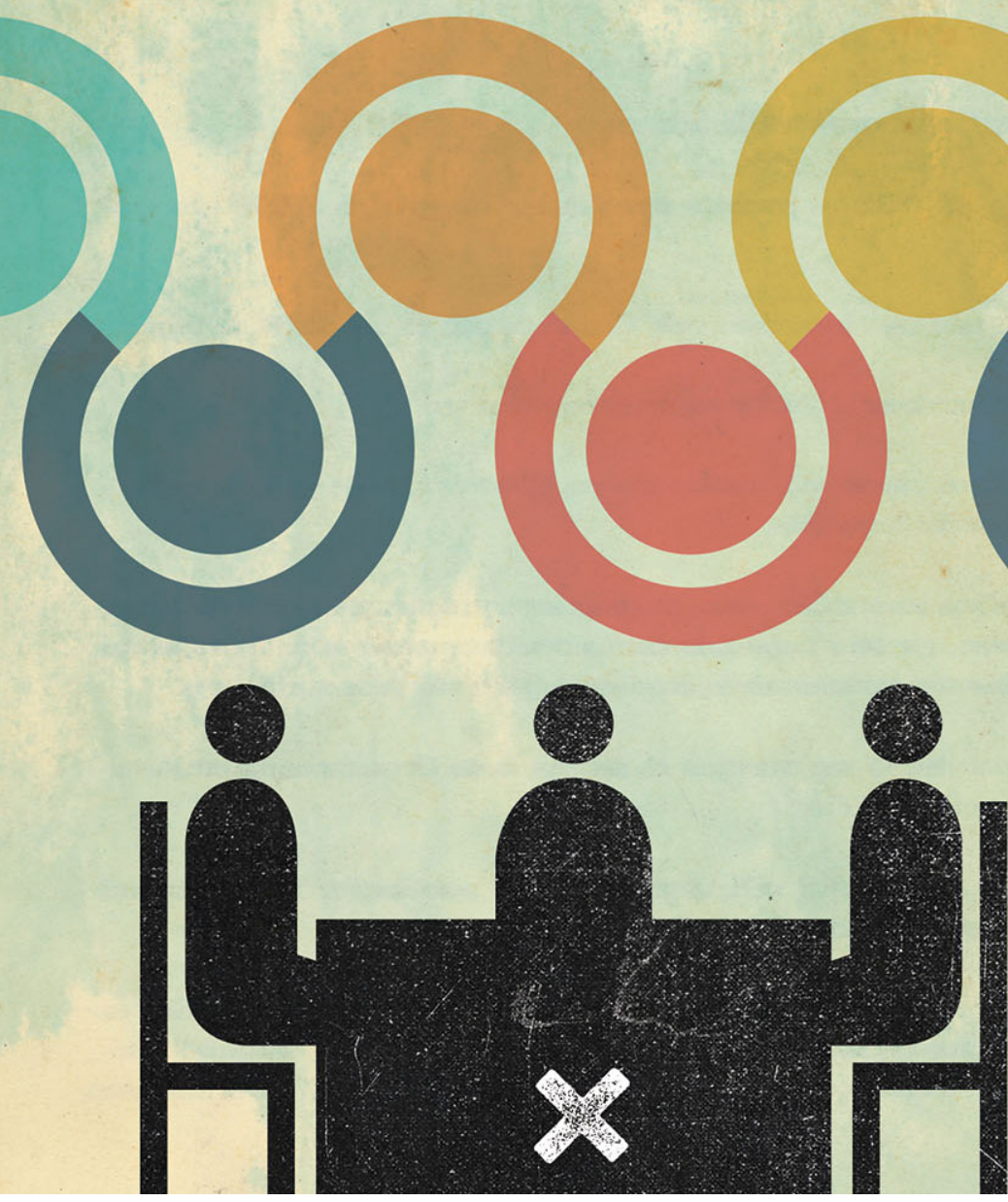
de 3,5 \% y una inflación cercana al 10\%. En 2016 se lleva a cabo la negociación colectiva y el cierre de convenios colectivos en la mayoría de los sectores de actividad. Por lo tanto, ante la situación económica del país y la postura del gobierno de no modificar los lineamientos salariales presentados a los representantes de los trabajadores y su rechazo por parte del PIT-CNT, por entender que la propuesta del Poder Ejecutivo no promueve el aumento del salario real, es de esperar un crecimiento de la conflictividad laboral.

A su vez, considerando la relativa consolidación de la negociación colectiva se debe avanzar en otras materias. Durante el gobierno de Mujica se propuso ajustes salariales anuales según la evolución de la productividad y vinculados a indicadores según los desempeños sectoriales y macroeconómicos dentro de los rangos meta de la inflación estimada por el Banco Central. Sin embargo, la iniciativa tuvo escaso éxito entre los empresarios y el movimiento sindical, dada la dificultad de recabar la información y su fiabilidad, la escasa representatividad de los indicadores de desempeño y la complejidad de las fórmulas en su uso práctico durante las negociaciones. Por ende, es importante la constitución de un sistema de información que me permita la mejor toma de decisiones considerando la heterogeneidad sectorial e intersectorial para una mejor vinculación entre la productividad y los aumentos salariales. Asimismo, aun con avances en estos diez años, se debería profundizar en despegar los temas predominantemente salariales del contenido de las negociaciones colectivas, aumentando la incidencia de otros como: la capacitación y la formación laboral, las cláusulas de género, las de seguridad y salud laboral, las de fomento del empleo juvenil, sobre todo este último punto, vinculándolo a la reciente aprobación de la ley de empleo juvenil.

Los nuevos arreglos neocorporativos otorgan cierta paridad en las negociaciones entre empresarios y trabajadores, sobre todo, allí donde las relaciones laborales son sumamente subordinadas (servicio doméstico y sector rural) o donde la organización sindical cuenta con escasa representación y representatividad (micro y pequeñas empresas). En otro sentido, el criterio de universalidad de los convenios colectivos puede conllevar a resultados duales. Permite que en el presente la tasa de cobertura de la negociación colectica alcance actualmente al $90 \%$ de los asalariados, pero el carácter sectorial y el principio de eficacia general erga omnes de los convenios colectivos, al homogeneizar realidades heterogéneas, también conllevan riesgos. Al PIT-CNT le supone un clivaje insiders-outsiders a resolver. Los dirigentes y trabajadores sindicalizados (insiders), negocian y acuerdan sobre cuestiones que afectarán a los no sindicalizados (outsiders), favoreciendo la aparición de acciones free riders dejando en manos del sindicato los riesgos, costos y el desgaste de las negociaciones, porque aun siendo mínima su ganancia, para los outsiders los costes de transacción serán más bajos. Esto a futuro, podría ser un límite a los incentivos para incorporar más trabajadores a las filas de los sindicatos (Carracedo, 2010) ${ }^{8}$. Por otra parte, a pesar de lograrse niveles relativamente bajos de informalidad (como no registro a la seguridad 


\title{
social), en la actualidad afecta a un cuarto de los ocupados, por lo que la negociación colectiva podría inducir la dualización y asentar la segmentación laboral entre quienes estén o no cubiertos por los términos de los convenios colectivos.
}

\begin{abstract}
Notas
1 La creación y reglamentación del CEN, por Ley № 17.935 y previsto por la Constitución de 1934 (Art. 206 y 207), lo formalizó como órgano privado de interés público, consultivo y honorario. Su integración es de: 14 representantes de los trabajadores (12 por los activos y dos por los pasivos); 14 por los empresarios (12 por los industriales, comerciales, agropecuarios y de otros servicios, y dos por los "empresarios pasivos"), tres de los cooperativistas; tres de los profesionales universitarios; tres por los usuarios y consumidores; tres por las organizaciones no gubernamentales en convenios con el Estado. Por el Estado estarán representados siete Ministerios, las empresas públicas, la banca pública (Banco Central, Banco República y Banco de Seguros del Estado), el Banco de Previsión Social (BPS) y la Oficina de Planeamiento y Presupuesto (OPP).

${ }^{2}$ El Consejo del Desarrollo Económico y Social creado por el gobierno de Lula, actúa como agencia de consulta, un canal institucional de negociaciones entre la sociedad civil y el gobierno (Fleury, 2004).

${ }^{3}$ Dicho decreto derogó aquél que facultaba al Ministerio del Interior a proceder a la desocupación de los huelguistas de los locales de trabajo por simple solicitud del empleador

${ }^{4}$ Esta ley no regula aspectos vinculados a la organización y constitución de sindicatos o al derecho a huelga, sino que establece la universalidad de los fueros, sean dirigentes sindicales o simples trabajadores, durante o sin negociación colectiva y la nulidad de toda práctica antisindical en la relación al acceso o permanencia en un empleo. Asimismo, dispone el derecho a la licencia sindical, el uso de la cartelera sindical en los lugares de trabajo, facilitar el uso de locales y horarios, la retención de la cuota sindical, hasta la reposición y/o reinstalación del trabajador despedido o discriminado por su actividad sindical.

${ }^{5}$ Prueba de ello es la participación de los representantes de los trabajadores en: la Administración Nacional de Educación Pública (ANEP), en la salud dentro de la Administración Nacional de Servicios de Salud del Estado (ASSE), en la seguridad social en el Banco de Previsión Social (BPS) y a través de los Consejos de Salarios (CCSS). A lo que se agrega en el Instituto Nacional de Empleo y Formación Profesional (INEFOP) o el Instituto Nacional del Cooperativismo (INACOOP).

${ }^{6}$ La"cooperación" con el FA, abrió en el PIT-CNT la sublevación interna de los sectores más radicales -que no contaban con representación parlamentaria o cargos de gobierno - reivindicando su condición de clase y la autonomía partidaria del movimiento sindical, con fuertes cuestionamientos a la dirigencia del PIT-CNT y a la línea económica seguida por el gobierno.

${ }^{7}$ El Diálogo Nacional por el Empleo se estructuró en base a cinco ejes temáticos: a) el empleo y la formación profesional de los jóvenes; b) políticas para enfrentar la insuficiencia actual en materia de oferta laboral; c) adecuación entre las estructuras de calificación de la oferta y la demanda laboral; d) políticas de empleo para las distintas fases del ciclo económico, y; e) empleo y políticas a nivel micro y mesoeconómico.

${ }^{8}$ Es apresurado dar por sentado este supuesto en tan corto plazo de continuidad institucional de los CCSS. Sin embargo, en los casos europeos con más tradición de negociación colectiva se observa que según la OCDE, por ejemplo: en Francia el grado de cobertura de los convenios colectivos es casi del 90\%, pero en las últimas décadas la tasa de sindicalización no supera el 10\% (OCDE, 1997) (aun considerando que existen varias centrales sindicales lo que podría dispersar las negociaciones). Por otra parte, Suecia mantiene casi sin variaciones una tasa de sindicalización cercana al 80\%. En ello pesan ciertos incentivos: para que los beneficios de los convenios colectivos alcancen a los trabajadores deben estar sindicalizados, sumado a la responsabilidad de la administración y la prestación de bienes públicos sociales (los seguros por desempleo), por parte de los sindicatos.
\end{abstract}

\section{Bibliografía}

AIDT, T.; TZANNATOS, Z. Union and Collective Bargaining. Washington D.C.: The World Bank, 2002.

ALVES, G.; AMARANTE, V.; SALAS, G.; VIGORITO, A. La desigualdad del ingreso en Uruguay entre 1986 y 2009. Serie Documentos de Trabajo del Instituto de Economía, n. 3, 2012. 
AMABLE, B.; BARRÉ, R.; BOYER, R. Los sistemas de innovación en la era de la globalización. Buenos Aires: Miño y Dávila /CEIL-PIETTE/Trabajo y Sociedad, 2008.

AMABLE, B. Les cinq capitalismes: Diversité des systèmes économiques et sociaux dans la mondialisation. Paris: Seuil, 2003.

AMARANTE, V.; ARIM, R.; YAPOR, M. Desigualdad e informalidad en el Uruguay. In: AMARANTE, V.; ARIM, R. (eds.). Desigualdad e informalidad: un análisis de cinco experiencias latinoamericanas. Santiago de Chile: Libros de la CEPAL, n. 133, Comisión Económica para América Latina y el Caribe, 2015.

ANTÍA, F.; CASTILLO, M.; FUENTES, G.; MIDAGLIA, C. La renovación del sistema de protección uruguayo: el desafío de superar la dualización. Revista Uruguaya de Ciencia Política, v. 22, n. 2, p. 171-194, 2013.

AOKI, M. The contingent governance of teams: analysis of institutionnal complementary. International Economic Review, v. 35, n. 3, p. 657-676, 1994.

BACCARO, L. Labour, Globalization and Inequality: Are Trade Unions Still Redistributive? Discussion paper 192. DP/192/2008. Geneva: International Institute for Labour Studies, 2008.

BIZBERG, I. (Coord.). Variedades de Capitalismo en América Latina. Los casos de México, Brasil, Argentina y Chile. México: El Colegio de México, 2015.

BOSCHI, R.; GAITÁN, F. Empresas, capacidades estatales y estrategias de desarrollo en Argentina, Brasil y Chile, 2009. Disponible en: <http://neic.iesp.uerj.br/textos/renato-wkshpniteroi.pdf >. Acceso en: 8 marzo 2011.

BOYER, R. How and why capitalisms differ, MPIfG Discussion Paper 02/5. In: Max Planck Institute for the Study of Societies, 2005, Cologne, Disponible en: <www.mpifg.de/pu/mpifg_dp/dp05-4. pdf> Acceso en: 31agosto, 2007.

BOYER, R. Le lien salaire/emploi dans la théorie de la régulation. Autant de relations que de configurations institutionnelles, documents du CEPREMAP no 9814. 1998.

BRADLEY, D.; STEPHENS, J. D. Employement Performance in OCDE Countries. A test of Neoliberal and Instutionalist Hypotheses. Comparative Political Studies, v. 49, n. 12, p. 1486-1510, 2007.

BRADLEY, D.; HUBER, E.; MOLLER, S.; NIELSEN, F.; STEPHENS, J. D. Distribution and Redistribution in Postindustrial Democracies, World Politics, v. 55, p. 193-228, 2003.

BURDIN, G.; ESPONDA, F.; VIGORITO, A. Desigualdad y sectores de altos ingresos en Uruguay: un análisis en base a registros tributarios y encuestas de hogares para el período 20092011. Serie Documentos de Trabajo del Instituto de Economía, n. 6, 2014.

CARRACEDO, F; SENATORE, L. Las políticas laborales y las relaciones de trabajo durante el gobierno de José Mujica. In: BENTANCUR, N.; BUSQUETS, J. El decenio progresista. Las políticas públicas de Vázquez a Mujica. 17-41. Montevideo: Fin de Siglo. Instituto de Ciencia Política. 2016.

CARRACEDO, F.; SENATORE, L. La evolución de la institucionalidad laboral: aportes, dificultades y desafíos. In: Informe de Coyuntura. Política en tiempos de Mujica III, a un año de las elecciones nacionales. Montevideo: Instituto de Ciencia Política, Facultad de Ciencias Sociales y Estuario Editores, 2013.

CARRACEDO, F. La tercera etapa de los Consejos de Salarios. Las relaciones laborales en la arquitectura socialdemócrata, 2010 (Licenciatura en Ciencia Política). Facultad de Ciencias Sociales, Universidad de la República, Montevideo. 
COMISIÓN ECONÓMICA PARA AMÉRICA LATINA Y EL CARIBE. La hora de la igualdad. Brechas por cerrar, caminos por abrir. Trigésimo tercer período de sesiones, Brasilia, 30 de mayo a 1 de junio 2010. Santiago de Chile: CEPAL, 2010.

COMISIÓN ECONÓMICA PARA AMÉRICA LATINA Y EL CARIBE. Eslabones de la desigualdad. Heterogeneidad estructural, empleo y protección social. Santiago de Chile: CEPAL, 2012.

EBBINGHAUS, B. When labour and capital collude: the political economy of early retirement in Europe, Japan and the USA. The Program for the Study of Germany and Europe Working Papers Series, 2000.

ERMIDA URIARTE, O. El protagonismo de los Consejos de Salarios en el sistema laboral uruguayo. Revista Derecho Laboral, v. LI, n. 229, p. 27, enero - marzo, 2008.

ESPING-ANDERSEN, G.; KORPI, W. La política social de clase en el capitalismo de posguerra: Escandinavia, Austria y Alemania. In: GOLDTHORPE, J. H. (Comp.) Orden y Conflicto en el capitalismo contemporáneo. Estudios sobre economía política en los países de Europa Occidental. Colección Economía y Sociología del Trabajo. Madrid: Ministerio de Trabajo y Seguridad Social, 1991.

ESPING-ANDERSEN, G. Los tres mundos del Estado del Bienestar. España: Alfons el Magnanim, 1993.

FILGUEIRA, F. Welfare and Democracy in Latin America: The Development, Crises and Aftermath of Universal, Dual and Exclusionary Social States, Geneva: UNRISD, 2005.

FLEURY, S. La concertación y eficacia de la acción política: el Consejo del Desarrollo Económico y Social del gobierno de Lula. Revista de Administración Pública, v. 34, n. 110, p. 111-119. Eneroabril 2004. Disponible en: <http://www.juridicas.unam.mx/publica/librev/rev/rap/cont/110/art/ art11.pdf>. Acceso en 10 abr. 2010.

FREYSSINET, C. El desafío de las políticas del empleo en el siglo XXI: La experiencia recientes de los países de Europa Occidental. Buenos Aires: Miño y Dávila /CEIL-PIETTE/ Trabajo y Sociedad, 2007. GOUREVITCH, P. The Politics of Corporate Governance regulation. Yale Law Journal 112, n. 7, p. 1829-80, 2003. Disponible en: <http://www.yalelawjournal.org/pdf/112-7/GourevitchFINAL. pdf> Acceso en: 15 julio 2010.

HALL, P.; SOSKICE, D. Les variétés du capitalisme. L'Année de la Régulation. n. 6, p.47-124. , 2002.

HALL, P.; SOSKICE, D. Varieties of Capitalism. The Institutional Foundations of Comparative Advantage. Oxford: Oxford University Press, 2001.

IVERSEN, T; SOSKICE, D. Distribution and Redistribution. The Shadow of the nineteenth Century. World Politics v. 61, n. 3, p 438-86, 2009.

IVERSEN, T.; SOSKICE, D. Electoral Institutions and the Politics of Coalitions: Why Some Democracies Redistribute More than Others. American Political Science Review, v. 100, n. 2, p. 165-181, 2006.

KENWORTHY; PONTUSSON, J. Rising Inequality and the Politics of Redistribution in Affluent Countries. Perspectives on Politics, v. 3, n. 3, p. 449-471, 2005.

KORPI, W. Power resources and employer-centered approaches in explanations of welfare states and varieties of capitalism protagonists, consenters, and antagonists. World Politics v. 58, n. 2, p. 167-206, 2006.

KORPI, W. Welfare-State Regress in Western Europe: Politics, Institutions, Globalization, and Europeanization, Annual Review Sociology, v. 29, p. 589-609, 2003. 
KORPI, W.; PALME, J. New Politics and Class Politics in the Context of Austerity and Globalization: Welfare State Regress in 18 Countries, 1975-1995. American Political Science Review, v. 97, n. 3, p. 1-22, 2003.

MARTÍNEZ FRANZONI, J. Regímenes de bienestar en América Latina. Documento de trabajo, nº 11. Fundación Carolina, 2007.

MÉNDEZ, G.; SENATORE, L. La política laboral a mitad de camino. In: Política en tiempos de Mujica II: un balance en mitad del camino. Montevideo: Instituto de Ciencia Política, Facultad de Ciencias Sociales y Estuario Editores, 2012.

MINISTERIO de TRABAJO y SEGURIDAD SOCIAL. Quinta Ronda de Consejos de Salarios 2012 - 2013. Unidad de Evaluación y Monitoreo de las Relaciones Laborales y Empleo, MTSS, Abril 2014. Disponible en: <http://www.mtss.gub.uy/documents/11515/59add4c5-f7d4-45ca-b79a6d747e50fc35>. Acceso en: 24 abr. 2014.

ORGANIZACIÓN para la COOPERACIÓN y el DESARROLLO ECONÓMICOS. Perspectives de l'emploi de l'OCDE 1997. Disponible en: < http://www.oecd.org/fr/els/emp/ perspectivesdelemploidelocde1997.htm>. Acceso en: 15 ago. 2009.

PALIER, B. Les évolutions de la protection sociale en Europe. Contraintes institutionnelles, evolutions d'ensemble, strategies politiques. Fondation Jean-Jaurès, n. 13, 2005.

PERAZZO, I.; CABRERA, V.; CÁRPENA, C. Cumplimiento de los acuerdos alcanzados en los Consejos de Salarios en Uruguay entre 2007 y 2011. Serie Documento de Trabajo del Instituto de Economía, n. 10/13, 2013.

PIERSON, P. Sobrellevando la austeridad permanente. Reestructuración del Estado de Bienestar en las democracias desarrolladas. Revista Zona Abierta, n. 114/115, p. 43-119, 2006.

PONTUSSON, J.; RUEDA, D.; WAY, C. Comparative Political Economy of Wage Distribution:The Role of Partisanship and Labour Market Institutions. British Journal of Political Science, v. 32, p. 281-308, 2002.

ROSSI ALBERT, R. La nueva tutela de la libertad sindical: un reto a la judicatura laboral. Revista Derecho Laboral, v. XLIX, n. 222, p. 377, abril - junio, 2006.

RUEDA, D.; PONTUSSON, J. Wage Inequalities and Varieties of Capitalism. World Politics, v. 52, n. 3, p. 350-83, 2000.

SCHMIDT, V. A. L'etat, l'économie et la protection sociale aux États-Unis et en Europe. Critique internationale, n. 27, p. 83-107, 2005.

SCHNEIDER, B. R. Hierarchical Market Economies and Varieties of Capitalism in Latin America. Journal of Latin American Studies, v. 41, n.03, p 553-575, 2009.

SCHNEIDER, B. R.; KARCHER, S. Complementarities and continuities in the political economy of labour markets in Latin America. Socio-Economic Review, v. 8, n. 4, p. 623 -651, 2010.

SCHNEIDER, B. R.; SOSKICE, D. Inequality in developed countries and Latin America: coordinated, liberal and hierarchical systems. Economy and Society, v. 38, n. 1, p. 17, 2009.

SCHRÖDER, M. Integrating Welfare and Production Typologies: How Refinements of the Varieties of Capitalism Approach call for a Combination of Welfare Typologies. Journal of Social Policy, n. 38, p. 1943, 2009.

SOJO, C.; PÉREZ SÁINZ, J. P. Reinventar lo social en América Latina. In: Desarrollo Social en América Latina: temas y desafíos para las políticas públicas. Costa Rica: FLACSO, Facultad Latinoamericana de Ciencias Sociales, 2002, p. 13-62.

THELEN, K. Varieties of Liberalization and the New Politics of Social Solidarity. New York: Cambridge University Press, 2014. 
TRAXLER, F.; BRANDL, B. The Economic Effects of Collective Bargaining Coverage: A Cross-National Analysis. Geneva: International Labour Office; Global Union Research Network (GURN). ILO, 2009.

WALLERSTEIN, M. Wage-Setting Institutions and Pay Inequality in Advanced Industrial Societies. American Journal of Political Science, v. 43, n. 3, p. 649-80, 1999.

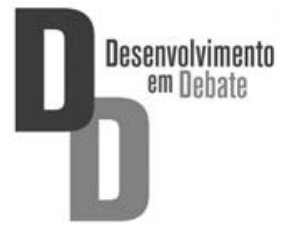

